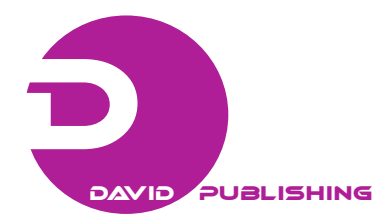

\title{
Mode Localization in Modular Trussed Structures and Subjected to Slightly Disordered Loading
}

\author{
Reyolando Manoel Lopes Rebello da Fonseca Brasil \\ Federal University of ABC, Santo André, SP 09210-170, Brazil
}

Received: December 31, 2012 / Accepted: January 29, 2013 / Published: March 25, 2013.

\begin{abstract}
Vibration modes localization in structures composed of several nominally identical lightly coupled modular substructures are studied. In an ideal perfect model, the vibration modes are global in nature, spreading to the whole structure. In real structures there are no two completely identical segments. Constructive or loading imperfections generate slight variation of the dynamic characteristics of each module. As the level of disorder grows and coupling between modules becomes lighter, the resulting vibration modes change considerably. Vibration energy may become confined to a few segments. This is the mode localization phenomenon. Models of long modular planar trussed structures are presented. Light coupling is considered between the initially identical modules. A certain degree of imperfection is introduced by adopting a slight variation in the loading of the modules. This will generate a small variation in the global stiffness of the system as the axial loads in the bars affect their geometric stiffness matrices.
\end{abstract}

Key words: Mode localization, modulated structures, trusses.

\section{Nomenclature}

$\begin{array}{ll}\text { A: } & \text { Transverse section area } \\ c: & \text { Cosine of } \alpha \\ E: & \text { Modulus of elasticity } \\ k: & \text { Bar stiffness matrix (GRF) } \\ k^{0}: & \text { Bar elastic stiffness matrix (GRF) } \\ k^{g}: & \text { Bar geometric stiffness matrix (GRF) } \\ \bar{k}: & \text { Bar stiffness matrix (LRS) } \\ \bar{k}^{0}: & \text { Bar elastic stiffness matrix (GRS) } \\ \bar{k}^{g}: & \text { Bar geometric stiffness matrix (GRS) } \\ \mathrm{K}: & \text { Structure stiffness matrix } \\ K_{\mathrm{ij}}: & \text { Stiffness coefficient } \\ L: & \text { Bar length } \\ \mathrm{m}: & \text { Bar mass matrix } \\ \mathrm{M}: & \text { Structure mass matrix } \\ N: & \text { Bar normal force } \\ P: & \text { Nodal load } \\ \mathrm{s}: & \text { Sine of } \alpha \\ \mathrm{T}: & \text { Rotation matrix }\end{array}$

Corresponding author: Reyolando Manoel Lopes Rebello da Fonseca Brasil, Ph.D., professor, research fields: dynamics and stability of structures. E-mail: reyolando.brasil@ufabc.edu.br.

\section{Greek Letters}

$\begin{array}{ll}\alpha: & \text { Angle } \\ \lambda: & \text { Eigenvalue } \\ \rho: & \text { Material density }\end{array}$

\section{Introduction}

In this paper, a study is presented of vibration modes localization in structures composed of several nominally identical lightly coupled modular substructures. In an ideal perfect model, the vibration modes are global in nature, spreading to the whole set of modular substructures. In real structures, on the other hand, there are no two completely identical segments. Constructive or loading imperfections generate slight variation of the dynamic characteristics of each module. As the level of disorder grows and coupling between modules becomes lighter, there is a possibility that the resulting vibration modes change considerably in relation to the ideal perfect model. Vibration energy may become confined to a few segments, even to only one of them, as opposed to the ordered case in which is spread over the whole structure. This is the so-called Mode Localization 
Phenomenon, first detected by Anderson [1], in the context of solid state physics.

In this paper, mathematical models are presented of long modular planar trussed structures. Light coupling is considered between the initially identical modules. A certain degree of imperfection is introduced in the system by adopting a slight variation in the nodal loading of the modules. This loading will generate a certain small variation in the global stiffness of the system as the axial loads in the bars affect their so-called geometric stiffness matrices or initial stress matrices.

Although the eigenvalue and eigenvector extraction process is a linear one, the consideration of the loading state of the structure on its stiffness is a result of a nonlinear effect, namely, that of the geometric stiffness. This is a new contribution to the state of art as compared to previous research by the author and collaborators [2-5].

\section{Formulation}

Here, a finite element formulation is presented of the element matrices of planar truss bar in local reference frame (LRF). First, the stiffness matrix is:

$$
\bar{k}=\bar{k}^{0}+\bar{k}^{g}
$$

where

$$
\bar{k}^{0}=\frac{E A}{L}\left[\begin{array}{cccc}
1 & 0 & -1 & 0 \\
0 & 0 & 0 & 0 \\
-1 & 0 & 1 & 0 \\
0 & 0 & 0 & 0
\end{array}\right]
$$

is the linear elastic stiffness matrix, $E$ is the material modulus of elasticity, $A$ is the cross section area, $L$ is the length of the bar, and:

$$
\bar{k}^{g}=\frac{N}{L}\left[\begin{array}{cccc}
0 & 0 & 0 & 0 \\
0 & 1 & 0 & -1 \\
0 & 0 & 0 & 0 \\
0 & -1 & 0 & 1
\end{array}\right]
$$

is the geometric stiffness matrix, where the axial force $N$ is positive if traction.

The planar truss bar stiffness matrix in global reference frame (GRF) is:

$$
k=T^{t} \bar{k} T
$$

where the rotation matrix is:

$$
T=\left[\begin{array}{cccc}
c & s & 0 & 0 \\
-s & c & 0 & 0 \\
0 & 0 & c & s \\
0 & 0 & -s & c
\end{array}\right]
$$

$c=\cos \alpha, s=\operatorname{sen} \alpha(\alpha$ is the angle between the bar and the GRF $x$-axis).

The resulting stiffness matrices in the GRF are:

$$
\begin{aligned}
k^{0} & =\frac{E A}{L}\left[\begin{array}{cccc}
C C & C S & -C C & -C S \\
S C & S S & -S C & -S S \\
-C C & -C S & C C & C S \\
-S C & -S S & S C & S S
\end{array}\right] \\
k^{g} & =\frac{N}{L}\left[\begin{array}{cccc}
S S & -S C & -S S & S C \\
-C S & C C & C S & -C C \\
-S S & S C & S S & -S C \\
C S & -C C & -C S & C C
\end{array}\right]
\end{aligned}
$$

A so-called consistent planar truss bar mass matrix is adopted:

$$
m=\frac{\rho A L}{6}\left[\begin{array}{llll}
2 & 0 & 1 & 0 \\
0 & 2 & 0 & 1 \\
1 & 0 & 2 & 0 \\
0 & 1 & 0 & 2
\end{array}\right]
$$

where $\rho$ is the density of the material. As it is well known, this matrix is invariant to rotation of reference frame.

\section{Results and Discussion}

As a first example, a two bay planar truss composed of equilateral triangles is presented, with sides of $L$ length, supported in the three bottom nodes. A very slender horizontal bar, providing light coupling, connects the upper nodes. Fig. 1 displays the adopted model.

The stiffness matrices for the bars inclined to the right are:

$$
k_{\text {incl, right }}^{0}=\frac{E A}{4 L}\left[\begin{array}{cccc}
1 & \sqrt{3} & -1 & -\sqrt{3} \\
\sqrt{3} & 3 & -\sqrt{3} & -3 \\
-1 & -\sqrt{3} & 1 & \sqrt{3} \\
-\sqrt{3} & -3 & \sqrt{3} & 3
\end{array}\right]
$$




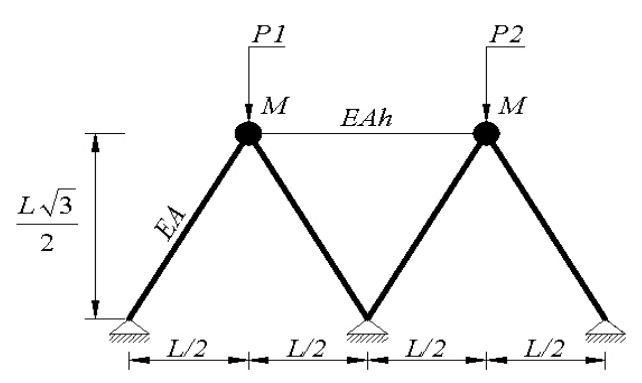

Fig. 1 The 2-degree-of-freedom model.

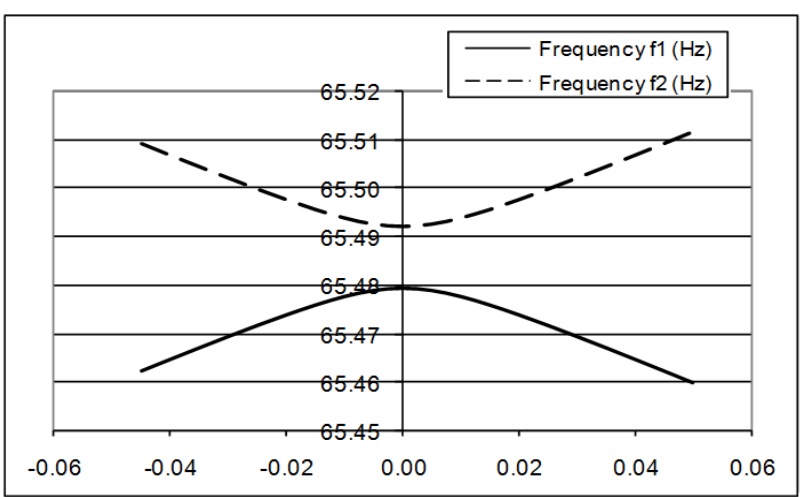

Fig. 2 Variation of frequencies 1 e 2 vs. $\varepsilon$.

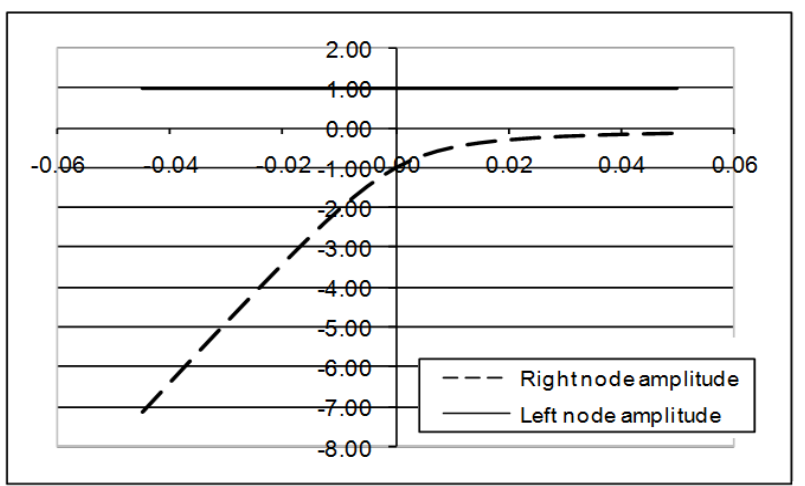

Fig. 3 Free vibrations relative amplitudes for 1st (anti-symmetrical) mode vs $\varepsilon$.

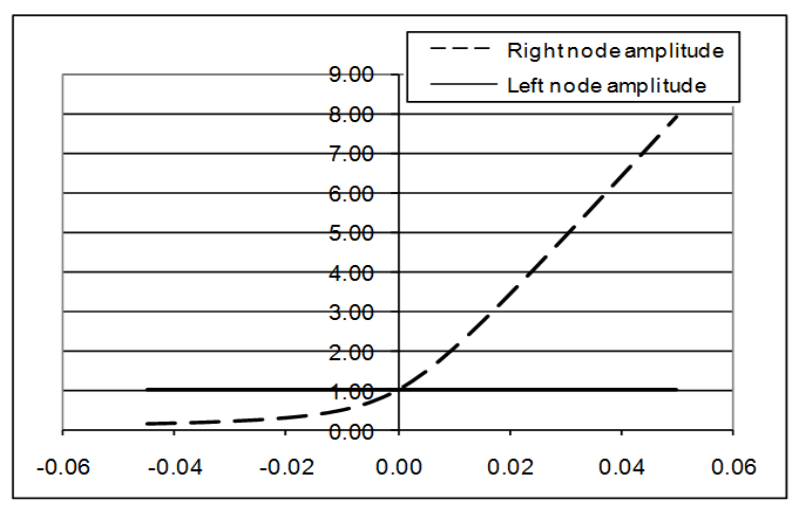

Fig. 4 Free vibrations relative amplitudes for 2nd (symmetrical) mode vs $\varepsilon$.

$$
k_{\text {incl, right } 1}^{g}=\frac{N}{4 L}\left[\begin{array}{cccc}
3 & -\sqrt{3} & -3 & \sqrt{3} \\
-\sqrt{3} & 1 & \sqrt{3} & -1 \\
-3 & \sqrt{3} & 3 & -\sqrt{3} \\
\sqrt{3} & -1 & -\sqrt{3} & 1
\end{array}\right]
$$

The stiffness matrices for the bars inclined to the left are:

$$
\begin{aligned}
& k_{\text {incl, left }} \mathbf{0}=\frac{E A}{4 L}\left[\begin{array}{cccc}
1 & -\sqrt{3} & -1 & \sqrt{3} \\
-\sqrt{3} & 3 & \sqrt{3} & -3 \\
-1 & \sqrt{3} & 1 & -\sqrt{3} \\
\sqrt{3} & -3 & -\sqrt{3} & 3
\end{array}\right] \\
& k_{\text {incl, left }}^{g}=\frac{N}{4 L}\left[\begin{array}{cccc}
3 & \sqrt{3} & -3 & -\sqrt{3} \\
\sqrt{3} & 1 & -\sqrt{3} & -1 \\
-3 & -\sqrt{3} & 3 & \sqrt{3} \\
-\sqrt{3} & -1 & \sqrt{3} & 1
\end{array}\right]
\end{aligned}
$$

In order to analyze the effect of load variation, the compressive axial forces in the bars used to compute the geometric matrices are calculated by equilibrium as functions of the vertical loads $P_{1}$ and $P_{2}$ (in down direction) applied to the two upper nodes:

$$
N_{i}=-\frac{P_{i} \sqrt{3}}{3}, \quad i=1,2
$$

The proposed variation of the loads is of the form:

$$
P_{1}=(1+\varepsilon) \bar{P} \quad P_{2}=(1-\varepsilon) \bar{P} \quad(-0.05 \leq \varepsilon \leq 0.05)
$$

The stiffness matrix for the horizontal connecting bar, is:

$$
k_{\text {horiz }}{ }^{0}=\frac{E A_{\text {horiz }}}{L}\left[\begin{array}{cccc}
1 & 0 & -1 & 0 \\
0 & 0 & 0 & 0 \\
-1 & 0 & 1 & 0 \\
0 & 0 & 0 & 0
\end{array}\right]
$$

The (symmetrical) global stiffness matrix for the four displacements of the two free upper nodes of the structure is:

$$
K=\left[\begin{array}{llll}
K_{11} & K_{12} & K_{13} & K_{14} \\
& K_{22} & K_{23} & K_{24} \\
& & K_{33} & K_{34} \\
\text { sym } & & & K_{44}
\end{array}\right]
$$

where

$$
K_{11}=\frac{E A+3 N_{1}}{2 L}+\frac{E A_{\text {horiz }}}{L}
$$




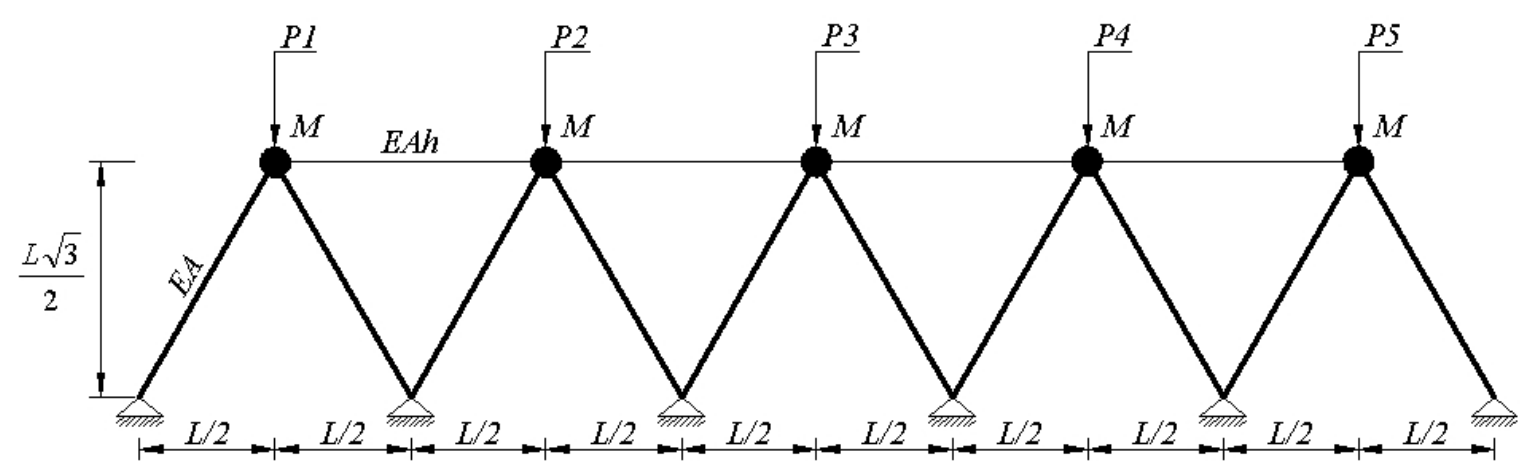

Fig. 5 The 5-degree-of-freedom model.

$$
\begin{gathered}
K_{33}=\frac{E A+3 N_{2}}{2 L}+\frac{E A_{\text {horiz }}}{L} \\
K_{13}=-\frac{E A_{\text {horiz }}}{L} \\
K_{12}=K_{14}=K_{23}=K_{24}=K_{34}=0 \\
K_{22}=\frac{3 E A+N_{1}}{2 L} \\
K_{44}=\frac{3 E A+N_{2}}{2 L}
\end{gathered}
$$

The corresponding global mass matrix is $\mathrm{M}$.

The sought circular frequencies $\omega$ are the solutions of the eingenvalue problem:

$$
\operatorname{det}|K-\lambda M|=0, \quad \lambda=\omega^{2} .
$$

In the numerical simulations the following numerical values were adopted: $E A=10^{9} \mathrm{~N}, E A_{\text {horiz }}=$ $10^{5} \mathrm{~N}, M=1,000 \mathrm{~kg}, L=3 \mathrm{~m}, \quad \bar{P}=9 \times 10^{6} \mathrm{~N}$.

Fig. 2 displays the variation with the degree of load imperfection $\varepsilon$ of the first two frequencies, $f_{1}$ related to the anti-symmetrical (solid line) and $f_{2}$ related to the symmetrical mode (dashed line). The so-called curve-veering phenomenon is clearly observed.

Fig. 3 displays the normalized horizontal amplitude of displacements for the two upper nodes for the first (anti-symmetrical) mode as function of the degree of load imperfection $\varepsilon$. Solid line indicates displacements for the left node and dashed line for the right node. Localization is clearly observed.

Fig. 4 displays the normalized horizontal amplitude of displacements for the two upper nodes for the second (symmetrical) mode as function of the degree of load imperfection $\varepsilon$. Solid line indicates displacements for the left node and dashed line for the right node. Localization is clearly observed.

Next, results are presented for a longer structure, displayed in Fig. 5, composed of five equilateral triangles similar to those presented in the first example.

Table 1 Randomly chosen $\beta$ coefficients in Eq. (24).

\begin{tabular}{llllll}
\hline Node $\mathrm{i}$ & 1 & 2 & 3 & 4 & 5 \\
\hline$\beta$ & 0.010 & -0.028 & 0.016 & -0.024 & -0.017 \\
\hline
\end{tabular}

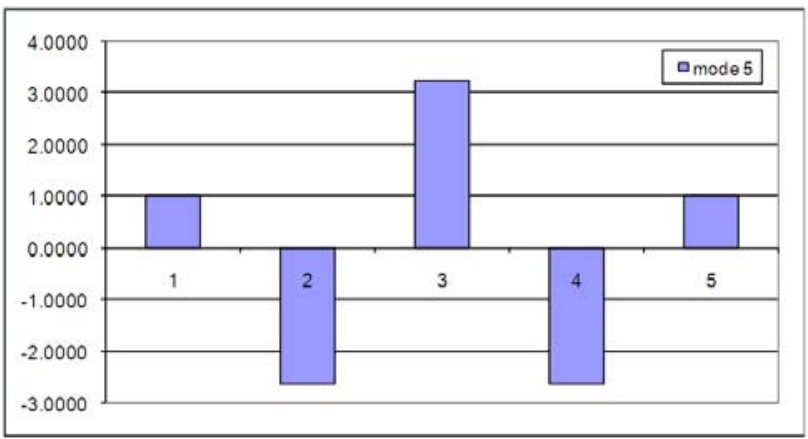

(a)

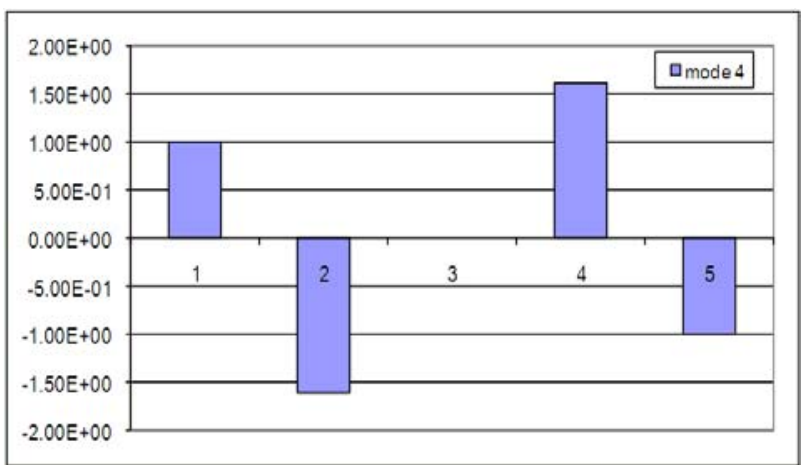

(b) 


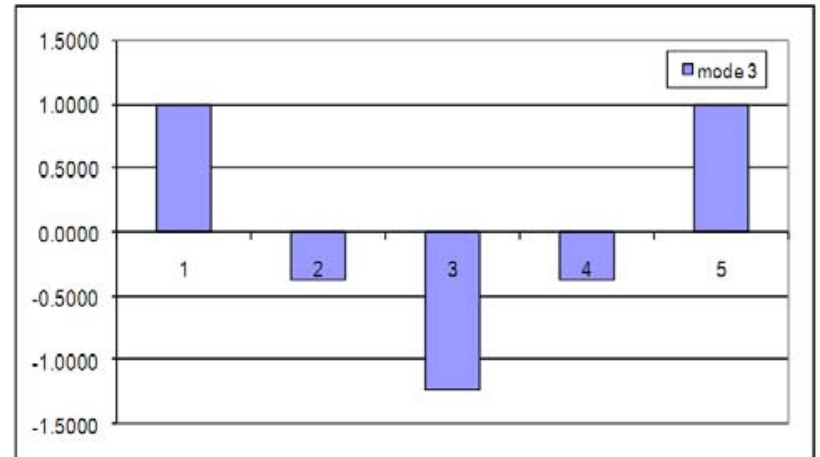

(c)

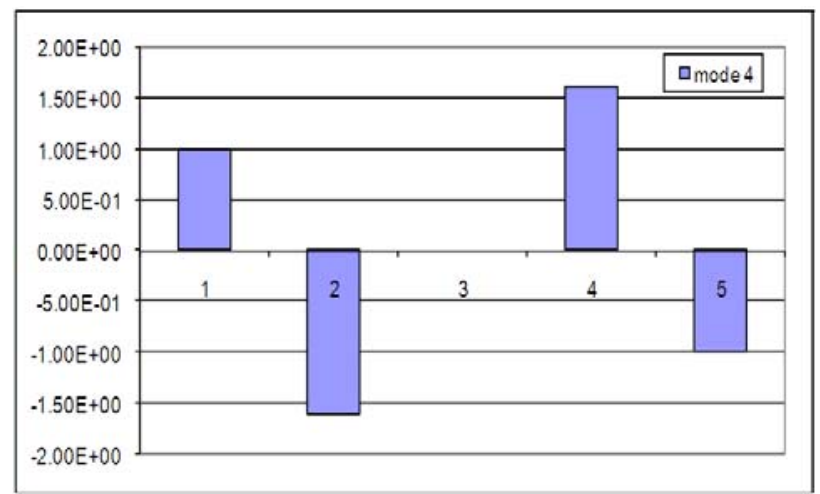

(d)

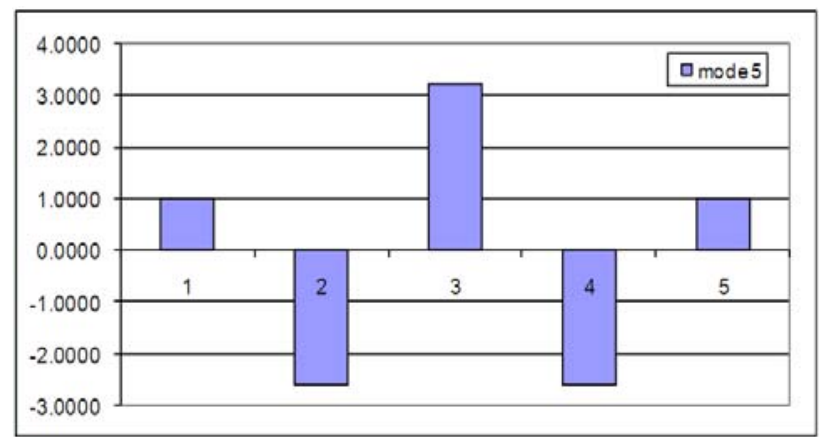

(e)

Fig. 6 (a) First mode, perfect mode, (b) second mode, perfect model, (c) third mode, perfect model, (d) forth mode, perfect model and (e) fifth mode, perfect model.

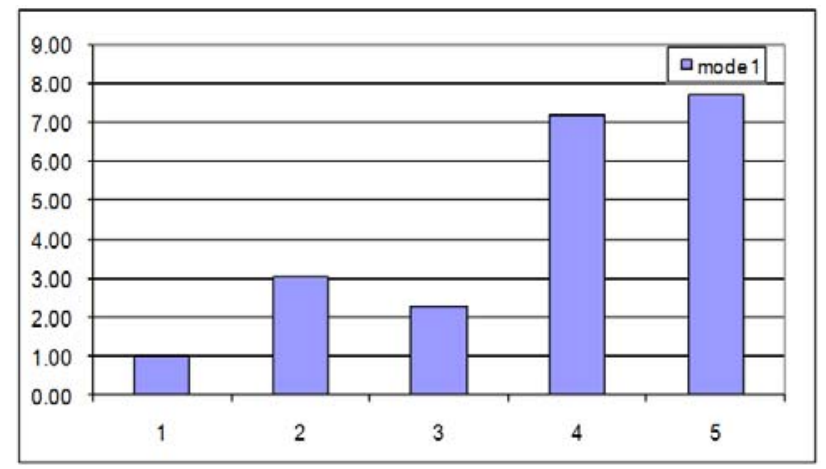

(a)

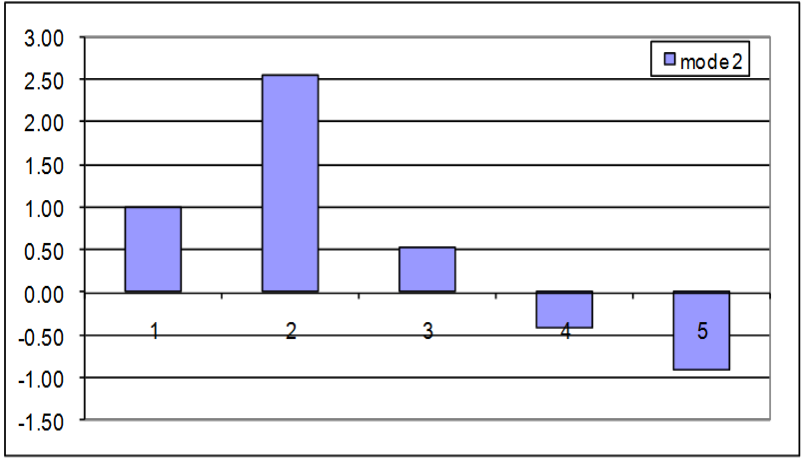

(b)

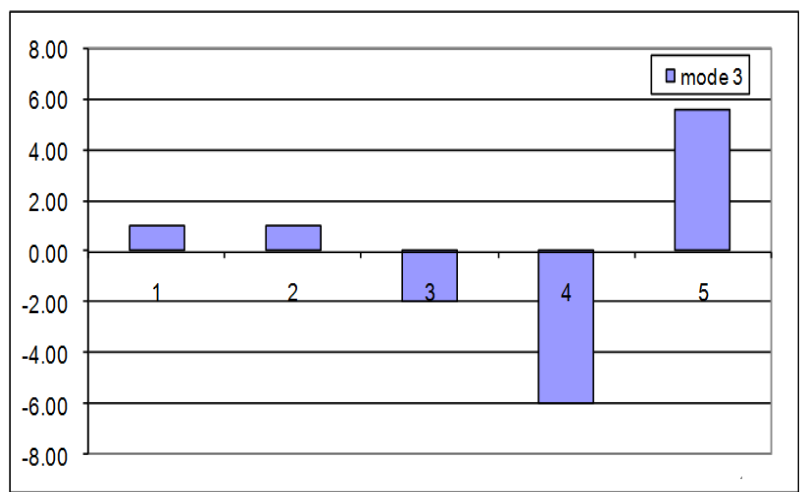

(c)

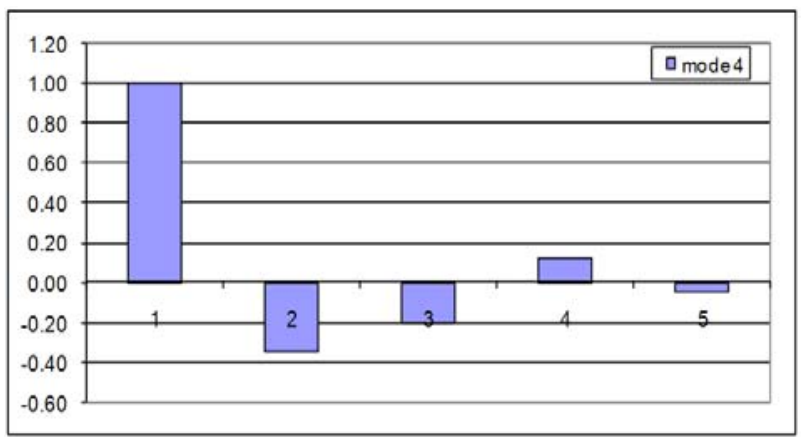

(d)

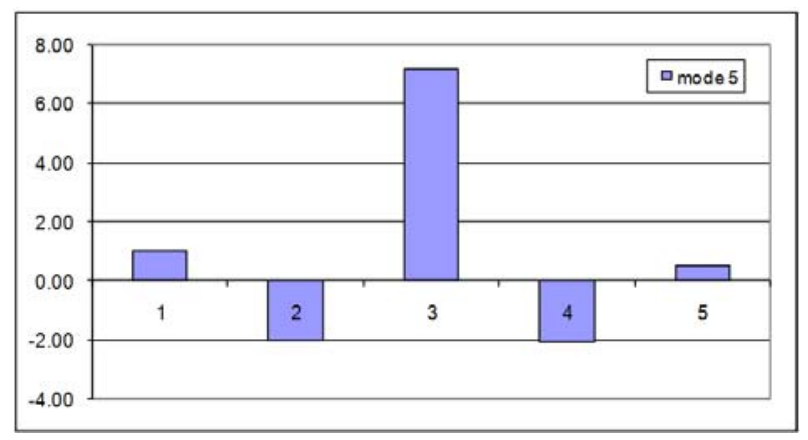

(e)

Fig. 7 (a) First mode, imperfect model, (b) second mode, imperfect model, (c) third mode, imperfect model, (d) forth mode, imperfect model and (e) fifth mode, imperfect model. 
In order to detect the influence of lightly disordered loading on the vibration modes, the 5 modes of the perfect model are presented, in Figs. 6a-6e, normalized in such way as to make the horizontal displacement of the fist mass equal to one.

Next, a certain small variation of the loads applied to the upper nodes is imposed in the form:

$$
P_{i}=\left(1+\beta_{i}\right) \bar{P}
$$

where the randomly chosen $\beta_{\mathrm{i}}$ multiplier are presented in Table 1.

For this imperfect loading the resulting 5 localized modes of the structure are prompted in Figs. 7a to 7e, normalized in such way as to make de horizontal displacement of the fist mass equal to one.

If one compares each mode of the imperfect model to the corresponding mode of the perfect model, mode localization is very clearly present, even for such the small amount of imperfections in the loading given by Table 1.

\section{Conclusions}

Long periodic planar trusses composed of many nominally identical lightly connected substructures were studied. The vibration modes for the perfect model of this kind of systems are usually global in nature, extended to the whole structure. If some small imperfections are present, the modes become localized. It is shown, in the examples, that even if the structure itself is perfectly periodic, small imperfections in the loading may cause mode localization as it influences the geometric stiffness of the mathematical model.

\section{Acknowledgments}

Support is acknowledged by FAPESP, CNPq and CAPES, all Brazilian research funding agencies.

\section{References}

[1] P.W. Anderson, Absence of diffusion in certain random lattices, Phys. Rev. 109 (1958) 1492-1505.

[2] J. Balthazar, R. Brasil, On nonlinear normal modes of a 2-dof model of a structure with quadratic nonlinearities, Journal of Sound and Vibration 182 (1995) 659-664.

[3] R. Brasil, M. Hawwa, The localization of buckling modes in nearly periodic trusses, Computers and Structures 56 (1995) 927-932.

[4] R. Brasil, C. Mazzilli, Influence of loading on mode localization in periodic structures, Applied Mechanics Reviews 48 (1995) 132-137.

[5] M. Hawwa, R. Brasil, Vibration confinement in trusses, Journal of Engineering Mechanics-ASCE 122 (1996) 286-290. 